\title{
A possible etiology of Kawasaki Disease
}

\section{Kimihiko Okazaki* \\ Okazaki Medical Clinic, Ukyoku, Kyoto, Japan}

According to Marian E. Melish, Kawasaki disease was first reported by Samuel Gee in 1871 [1], although the disease was not described as "infantile periarteritis nodosa" at that time. Tomisaku Kawasaki gave a remarkable delineation of the clinical features of the disease in 1967 [2]. In spite of the fact that a great number of out-standing pathologists as well as pediatricians have researched, the disease's etiology has not been demonstrated.

Apart from the fact mentioned above, the author of this short essay came across a fact that repeated intradermal injections with a nonspecific antigen preparation bring about a complete recovery from bronchial asthma in 1989. I was ignorant of the action-mechanism of the above-mentioned fact until the beginning of April, 1991 when I was informed of a theory proposed by Sohmei Kojima [3]. The theory was as follows: The reason why few parasite carriers suffer from allergic diseases can be because anti-parasite antibodies pre-occupy antibodyreceptors on the surface of the carriers' mast cells. He proposed the above theory in order to explain the experience of JA Turton that a 3-time repetition of infection and extermination of hookworms offering himself as a testee brought about a complete recovery from his 30-year-lasting pollen allergy [4]. Namely, I realized that the nonspecific antigen preparation had played the role of parasites. In other words, I realized that antibodies could exchange themselves on their receptors.

As far as the etiology of Kawasaki disease is concerned, it may not appear that there is some relationship between them, i.e., the disease's etiology and the antibodies' potentiality to exchange mutually. However, in my opinion, there does exist a relationship, of which bases are as follows: (1) It is well known that incidence rate of Kawasaki disease is remarkably high in Japan. (2) Infusion of gamma-globulin is a considerably effective treatment of the disease. (3) Infants' immunity does not establish until they are 6 months old. (4) The Japanese people tend to take hot baths more often than other nations. Consequently, non-pathogenic microorganisms on the body-surface of Japanese people must be quantitatively less than those of other nations. and (5) Causes of physical harm are classified as physical stimuli, chemical stuffs, microorganisms, and immunological (autoimmune and allergic) destructive actions.

In conclusion, Japanese infants are more susceptible of immunological destructive actions than infants of other nations. In conclusion, again, the extremely hygienic environment of Japanese infants is responsible for their high incident rate to suffer from Kawasaki disease. Needless to say, longer intervals to take hot baths may reduce the number of patients of Kawasaki disease. As medical treatments, intradermal injections with a non-specific antigen preparation are recommended. Although no evidence is available, yet, patients of Kawasaki disease may have an abnormally large quantity of anti-arterial antibody.

\section{References}

1. Gee S (1871) Aneurysms of coronary arteries in a boy. St Barth Hosp Rep 7: 14

2. Kawasaki T (1967) Acute febrile mucocutaneous syndrome with lymphoid involvement with specific desquamation of the fingers and toes in children. Japanese Journal of Allergology 16: 178-222.

3. Kojima S (1979) Parasite diseases, IgE and IgE antibodies. Rinsho-I 5: 679.

4. Turton JA (1976) IgE, Parasites, and allergy. Lancet 2: 686.
Copyright: (C2017 Okazaki K. This is an open-access article distributed under the terms of the Creative Commons Attribution License, which permits unrestricted use, distribution, and reproduction in any medium, provided the original author and source are credited.
Correspondence to: Kimihiko Okazaki, Okazaki Medical Clinic, Ukyoku, Kyoto, Japan,E-mail: ma13081x@ma1.seikyou.ne.jp

Received: February 14, 2017; Accepted: February 27, 2017; Published: February 28, 2017 